\title{
Strong "Quantum" Chaos in the Global Ballooning Mode Spectrum of Three-Dimensional Plasmas
}

\author{
R. L. Dewar* \\ Princeton University Plasma Physics Laboratory, P.O. Box 451, Princeton, New Jersey 08543 \\ P. Cuthbert and R. Ball \\ Department of Theoretical Physics and Plasma Research Laboratory, Research School of Physical Sciences \& Engineering, \\ The Australian National University, Canberra 0200, Australia
}

(Received 27 November 2000)

\begin{abstract}
The spectrum of ideal magnetohydrodynamic (MHD) pressure-driven (ballooning) modes in strongly nonaxisymmetric toroidal systems is difficult to analyze numerically owing to the singular nature of ideal MHD caused by lack of an inherent scale length. In this paper, ideal MHD is regularized by using a $k$-space cutoff, making the ray tracing for the WKB ballooning formalism a chaotic Hamiltonian billiard problem. The minimum width of the toroidal Fourier spectrum needed for resolving toroidally localized ballooning modes with a global eigenvalue code is estimated from the Weyl formula. This phase-space-volume estimation method is applied to two stellarator cases.
\end{abstract}

DOI: 10.1103/PhysRevLett.86.2321

PACS numbers: 52.35.Py, 05.45.Mt, 52.55.Hc

In design studies for new magnetic confinement devices for fusion plasma experiments (e.g., investigations $[1,2]$ leading to the proposed National Compact Stellarator Experiment, NCSX [3]), the maximum pressure that can stably be confined in any proposed magnetic field configuration is routinely estimated by treating the plasma as an ideal magnetohydrodynamic (MHD) fluid. One linearizes about a sequence of equilibrium states with increasing pressure, and studies the spectrum of normal modes (frequency $\omega$ ) to determine when there is a component with $\operatorname{Im} \omega>0$, signifying instability.

Even with the simplification obtained by using the ideal MHD model, the computational task of determining the theoretical stability of a three-dimensional (i.e., nonaxisymmetric) device, such as NCSX or the four currently operating helical axis stellators [4], remains a challenging one.

The problem can be posed as a Lagrangian field theory, with the potential term being the energy functional $\delta W$ [5]. For a static equilibrium, the kinetic energy is quadratic in $\omega$, so that $\omega^{2}$ is real. Thus instability occurs when $\omega^{2}<0$. There are two main approaches to analyzing the spectrum-local and global.

In the local approach, which is used for analytical simplification, one orders the scale length of variation of the eigenfunction across the magnetic field lines to be short compared with equilibrium scale lengths [6]. Both interchange and ballooning stability can be treated by solving the general ballooning equations [7], a system of ordinary differential equations defined on a given magnetic field line.

The global (Galerkin) approach is to expand the plasma displacement field in a finite basis set, inserting this ansatz in the Lagrangian to find a matrix eigenvalue representation of the spectral problem. This approach has been im- plemented for ideal MHD in three-dimensional plasmas in two codes, TERPSICHORE [8] and CAS3D [9].

Although the Galerkin approach is potentially exact, if one could use a complete, infinite basis set, it is in practice computationally challenging due to the large number of basis functions required to resolve localized instabilities. This leads to very large matrices which must be diagonalized by iterative methods. There is a need for analytical insight to determine a suitable truncated basis set and to predict the nature of the spectrum, e.g., whether it is continuous or discrete.

Such insight may be obtained by a hybrid local-global approach, in which one uses a Wentzel-Kramers-Brillouin (WKB) representation of the eigenfunction. In the shortwavelength limit, the same analytical simplifications as are obtained in the local approach are found to give a local dispersion relation that can be used to give information on the global spectrum by using ray tracing and semiclassical quantization.

In axisymmetric systems [10] or in cases where helical ripple can be averaged out, giving an adiabatic invariant $[11,12]$, the ray equations are integrable and hence the spectrum is characterized by "good quantum numbers."

However, it has been known for many years [7] that the ray-tracing problem in strongly three-dimensional systems is singular because, in the absence of an adiabatic invariant, the phase-space motion is not bounded-the rays escape to infinity in the wave vector sector. Dewar and Glasser [7] argued that this gives rise to a continuous unstable spectrum, with correspondingly singular generalized eigenfunctions. (A more rigorous treatment involves the concept of the essential spectrum and Weyl sequences $[13,14]$.)

Our proposed regularization of this singularity can be understood using a simple quantum analogy. Consider 
the one-dimensional time-independent Schrödinger equation $H \psi=E \psi$ in the limit as the mass of the particle goes to infinity. Then the kinetic energy disappears and the Hamiltonian becomes $H=V(x)$, where $V$ is the potential energy, assumed here to be the harmonic oscillator potential, $\frac{1}{2} x^{2}$ in suitable units. In the usual Hilbert space the energy spectrum is continuous: $E \geq 0$ and the (generalized) eigenfunctions singular: $\psi(x)=\delta\left(x-x_{\mathrm{E}}\right) \pm$ $\delta\left(x+x_{\mathrm{E}}\right)$, where $V\left(x_{\mathrm{E}}\right) \equiv E$.

We now seek a regularization of this problem by restricting $\psi$ to the space of functions with a finite bandwidth in wave number $k$ :

$$
\psi(x)=\int_{-k_{\max }}^{k_{\max }} \frac{d k}{2 \pi} \psi_{k} \operatorname{expikx}
$$

This truncated Fourier-integral representation models what occurs when one seeks to find the spectrum numerically using a truncated Fourier-series representation.

We take as a starting point a Lagrangian for the wave function,

$$
L=\int_{-\infty}^{\infty} \psi^{*}[E-V(x)] \psi d x
$$

Inserting Eq. (1) in Eq. (2) gives

$$
\begin{gathered}
L=\int_{-k_{\max }-0}^{k_{\max }+0}\left[E\left|\psi_{k}\right|^{2}-\mid \frac{d \psi_{k}}{d k}+\psi_{k} \delta\left(k+k_{\max }\right)\right. \\
\left.-\left.\psi_{k} \delta\left(k-k_{\max }\right)\right|^{2}\right] \frac{d k}{2 \pi}
\end{gathered}
$$

This is infinite unless we require the coefficients of the $\delta$ functions to vanish. That is, $\psi_{k}=0$ at $k= \pm k_{\max }$. The Euler-Lagrange equation is $\left(d^{2} / 2 d k^{2}+E\right) \psi_{k}=0$, which has the solutions $\exp \pm i(2 E)^{1 / 2} k$. These waves would propagate to infinity if it were not for the reflecting boundary conditions at $\pm k_{\max }$ we have just derived.

That is, we have removed the continuum by box quantization in $k$ space. In the following we shall do the same for the ballooning mode problem.

As in [7] we write the magnetic field of an arbitrary three-dimensional toroidal equilibrium plasma with nested magnetic flux surfaces labeled by an arbitrary parameter $s$ as $\mathbf{B}=\boldsymbol{\nabla} \zeta \times \nabla \psi-q \nabla \theta \times \nabla \psi \equiv \nabla \alpha \times \nabla \psi$, where $\alpha \equiv \zeta-q \theta$. Here, $\theta$ and $\zeta$ are the poloidal and toroidal angles, respectively, $\psi(s)$ is the poloidal flux function, and $q(s)$ is the inverse of the rotational transform. Since B . $\boldsymbol{\nabla} s=\mathbf{B} \cdot \boldsymbol{\nabla} \alpha=0, s$ and $\alpha$ serve to label an individual field line.

We take the stream function [6] to be given by $\varphi=$ $\hat{\varphi} \exp (i S-i \omega t)$, where $\hat{\varphi}(\theta \mid s, \alpha)$ is assumed to vary on the equilibrium scale. The phase variation is taken to be rapid, so $\mathbf{k} \equiv \nabla S$ is ordered to be large. The frequency $\omega$ is ordered $O(1)$, which requires that the wave vector be perpendicular to $\mathbf{B}: \mathbf{k} \cdot \mathbf{B} \equiv 0$. (In this study we consider unstable ideal MHD modes, $\omega^{2}<0$.)

It immediately follows that the eikonal is constant on each field line: $S=S(\alpha, s)$. From the definition of the wave vector, $\mathbf{k}=k_{\alpha} \nabla \alpha+k_{s} \nabla s \equiv k_{\alpha}[\nabla \alpha+$ $\left.\theta_{k} q^{\prime}(s) \nabla s\right]$, where $k_{\alpha} \equiv \partial S / \partial \alpha$ and $k_{s} \equiv \partial S / \partial s$. Here the anglelike ballooning parameter $\theta_{k}$ appears naturally as the ratio $k_{s} / q^{\prime}(s) k_{\alpha}$ [10].

The ballooning equation emerges in the large $|\mathbf{k}|$ expansion [6,7] as an ordinary differential equation to be solved on each field line $(\alpha, s)$ with given $\left(k_{\alpha}, k_{s}\right)$ under the boundary condition $\hat{\varphi}(\theta) \rightarrow 0$ at infinity to give the eigenvalue $\lambda\left(\alpha, s, k_{\alpha}, k_{s}\right)$. This constitutes a local dispersion relation $\lambda \equiv \rho \omega^{2}$ (the mass density $\rho$ being assumed constant everywhere).

The ray equations are the characteristics of the eikonal equation $\lambda\left(\alpha, s, \partial_{\alpha} S, \partial_{s} S\right)=\rho \omega^{2}$. These are Hamiltonian equations of motion with $\alpha, s$ the generalized coordinates, $k_{\alpha}, k_{s}$ the canonically conjugate momenta, and $\lambda$ as the Hamiltonian.

In axi- or helically symmetric systems all field lines on a given magnetic surface are equivalent $-\alpha$ is ignorable and $k_{\alpha}$ is a constant of the motion. In this case the equations are integrable and semiclassical quantization can be used to predict the approximate spectrum of global ballooning instabilities [10]. This technique can sometimes be applied successfully, even in nonsymmetric systems, if there are regions of phase space with a large measure of invariant tori $[11,15]$. In [11] this was verified using the global eigenvalue code TERPSICHORE [8].

At the other extreme, if the ray orbits are chaotic (but still bounded) then the global spectrum is not regularly structured, but must rather be described statistically by the density of states and the probability distribution of level spacings using the techniques of quantum chaos theory (see, e.g., $[16,17]$ ).

However, because of the scale invariance of the ideal MHD equations, $\lambda$ depends only on the direction of $\mathbf{k}$, not on its magnitude: $\lambda=\lambda\left(\alpha, s, \theta_{k}\right)$. This has the consequence that the ray orbits are unbounded in phase space, so, strictly speaking, ideal MHD gives rise to a quantum chaotic scattering $[16,17]$ problem rather than a straight quantum chaos problem. This leads to the continuous spectrum [7] with singular generalized eigenfunctions that cannot really be represented using the simple eikonal ansatz.

On the other hand, the absence of a natural length scale in ideal MHD is a mathematical artifact. Physically, the ion Larmor radius provides a lower cutoff in space, or an upper cutoff in $|\mathbf{k}|$, beyond which ideal MHD ceases to apply. The ballooning equation is also physically regularized by inclusion of diamagnetic drift $[15,18]$.

However, since in general it leads to a complex ray tracing problem [19], we shall not attempt to model diamagnetic drift stabilization in this paper. Rather, we regularize 
the ray equations simply by adding a barrier term to the effective ray "Hamiltonian" $H\left(\alpha, s, k_{\alpha}, k_{s}\right)$,

$$
H=\lambda\left(\alpha, s, k_{\alpha}, k_{s}\right)+U\left(k_{\alpha}\right),
$$

where the barrier potential we use is $U\left(k_{\alpha}\right) \equiv K\left(\left|k_{\alpha}\right|-\right.$ $\left.k_{\max }\right)^{2}$ for $\left|k_{\alpha}\right|>k_{\max }$ and 0 for $\left|k_{\alpha}\right|<k_{\max }$. In the limit of the constant $K \rightarrow \infty$, this infinite box potential gives the ideal MHD ray equations for $\left|k_{\alpha}\right|<k_{\max }$ and reflecting boundary conditions at $\left|k_{\alpha}\right|=k_{\max }$. Thus we have a twodegree of freedom Hamiltonian billiard problem.

Although overly crude for modeling FLR stabilization, the cutoff at $\left|k_{\alpha}\right|=k_{\max }$ provides a reasonable model for representing the finite spectral bandwidth in the toroidal Fourier mode number $(n)$ representation used in the global eigenvalue codes TERPSICHORE [8] and CAS3D [9].

Using ballooning-unstable plasma equilibria calculated for the H-1NF heliac $[4,20]$ using the VMEC code $[21]$, detailed parameter scans have been undertaken for two cases. The first case studied [22] was obtained by increasing the pressure gradient of a marginally stable equilibrium [23] uniformly across the plasma and thus was ballooning unstable at the edge of the plasma. The ray tracing problem for this case would involve consideration of the effect of the plasma boundary.

Thus a second equilibrium, ballooning stable near the edge of the plasma, was calculated for the purposes of the present paper. This case has a more peaked pressure profile than the first, but both have average $\beta \approx 1 \%$, where $\beta$ is the ratio of plasma pressure to magnetic field pressure.

The $q$ profiles are not monotonic - in the peaked pressure profile case studied in this paper, $q$ was 0.8895 on the magnetic axis, rising to a maximum value of 0.8964 quite close to the magnetic axis, then falling monotonically to 0.8675 . Clearly the (global) magnetic shear is very weak. Despite this fact and the nonmonotonicity, there is some formal simplification in choosing $s \equiv q$, and we have taken $s=q$ since the region of plasma studied is in a monotonic-decreasing part of the $q$ profile (the decreasing region outside the maximum- $q$ surface).

In these scans the most unstable ballooning eigenvalue was tabulated on a three-dimensional grid in $s, \alpha, \theta_{k}$ space. The dependence on $\alpha$ was found to be rapid. The dependence on $\theta_{k}$ was much slower, but the variation was sufficient that the higher-growth-rate isosurfaces formed a set of distinct, topologically spherical branches. It was argued in [22] that this branch structure is produced by Anderson localization in bad curvature regions due to the strong breaking of both helical and axisymmetry in H-1NF.

According to the perturbation expansion in $q^{\prime}$ described in [22], a quadratic form in $\alpha, \theta_{k}$ should form a good approximation to $\lambda-\lambda_{\min }(q)$ in the neighborhood of the central branch. Accordingly a least-squares fit on each surface was performed to provide a simple analytical description of the $(0,0)[22]$ branch.
The radial dependence of the fitting coefficients was approximated by fitting to third-degree polynomials in $q$. Sections of the resulting approximation to the central branch are shown in Fig. 1. The isosurface spans a substantial range of magnetic surfaces within the plasma - the narrow range of variation in $q$ is due to the low magnetic shear in $\mathrm{H}-1 \mathrm{NF}$.

In order to establish the nature of the ray dynamics described by the regularized Hamiltonian, Eq. (4), a numerical integration with cutoff at $k_{\max }=50$ was performed with initial conditions $q=q_{2}, \alpha=0$, and $k_{\alpha}=5$, where $\left[q_{1}, q_{2}\right]=[0.8852,0.8951]$ is the $q$ range spanned by the $\lambda=-6$ isosurface as seen in Fig. 1. (A run with $k_{\alpha}=10$ was also performed, with similar results.) Choosing the value $K=1$ gave a good compromise between the sharp boundary potential to be modeled, and the smooth potential required for the numerical integration. The orbit remained on the "energy shell" $\lambda=-6$ to within an accuracy of one part in $10^{6}$ over the "time" interval of the integration, 7500 .

The two Poincaré plots in Fig. 2 show the orbit to be strongly chaotic, filling the "energy shell" ergodically, except that the regions $k_{\alpha}>0$ and $k_{\alpha}<0$ are dynamically disjoint. The solid curve shown surrounding the outer limits of the "energetically accessible" region is calculated by solving $\lambda\left(0, q, k_{q} / k_{\max }\right)=-6$.

According to the Weyl formula [16], the number, $N\left(\lambda_{\max }\right)$, of global eigenmodes with eigenvalues below the eigenvalue $\lambda_{\max }$ is given, asymptotically in the limit $N \rightarrow$ $\infty$, as $N\left(\lambda_{\max }\right) \sim v_{4 \mathrm{D}}\left(\lambda_{\max }\right) /(2 \pi)^{2}$. Here $v_{4 \mathrm{D}}\left(\lambda_{\max }\right)$ is the volume of the dynamically accessible four-dimensional phase-space region $\lambda\left(\alpha, q, k_{q} / k_{\alpha}\right)<\lambda_{\max }, 0<k_{\alpha}<$ $k_{\max }$. The $k_{\alpha}$ integration can be performed analytically, giving $v_{4 \mathrm{D}}\left(\lambda_{\max }\right)=\frac{1}{2} k_{\max }^{2} v_{3 \mathrm{D}}\left(\lambda_{\max }\right)$, where $v_{3 \mathrm{D}}\left(\lambda_{\max }\right)$ is the volume within the isosurface $\lambda\left(\alpha, q, \theta_{k}\right)=\lambda_{\max }$. Thus

$$
N\left(\lambda_{\max }\right) \sim \frac{1}{8 \pi^{2}} k_{\max }^{2} v_{3 \mathrm{D}}\left(\lambda_{\max }\right) .
$$

We can make a rather rough estimate of the minimum value of $n_{\max }$ required for CAS3D or TERPSICHORE to find
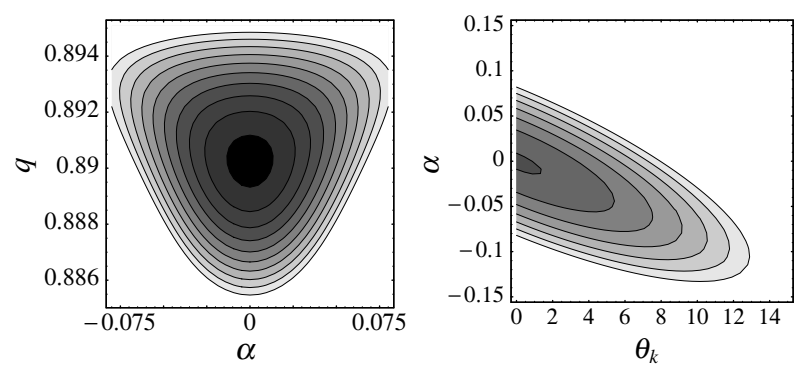

FIG. 1. The sections $\theta_{k}=0$ and $q=0.893$ of the topologically spherical isosurfaces of the central, $(0,0)$, ballooning mode branch, bounded by the isosurface $\lambda=-6$ (arbitrary units). The darker shades denote higher growth rates, the peak corresponding to $\lambda \approx-8$. 

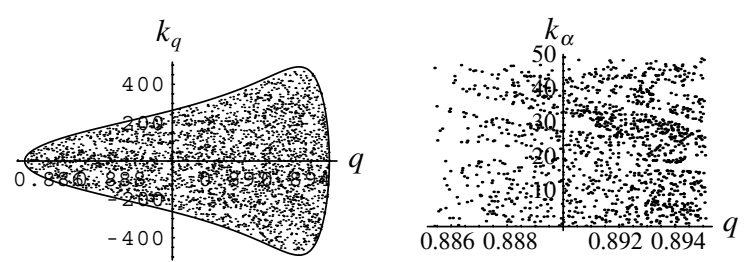

FIG. 2. Two views of intersections with the Poincaré surface of section $\alpha=0$.

even one eigenvalue with $\lambda<\lambda_{\max }$ by setting $N\left(\lambda_{\max }\right)=$ 1 and calculating $k_{\max } \approx n_{\max }$ from Eq. (5). This gives $n_{\max }(N=1) \sim\left(8 \pi^{2} / v_{3 \mathrm{D}}\right)^{1 / 2}$.

The isosurface $\lambda=-6$ studied above is about the largest of the disjoint topologically spherical isosurfaces corresponding to the highly toroidally localized strongly ballooning unstable regions of $\alpha, q, \theta_{k}$ space. (For $\lambda>-6$ the isosurfaces are no longer topologically spherical.) Using the polynomial fits described above, we calculate $v_{3 \mathrm{D}}(-6)=0.02158$. This gives $n_{\max }(N=1) \approx 60$. Assuming that the dominant contributions to the MHD energy $\delta W$ come from the rational surfaces intersecting the $\lambda=-6$ isosurface, we thus predict that it would be necessary to include, as a minimum set, basis functions corresponding to one of the two "mode families" [9] contained in the set $(n, m)=(9,8),(18,16)$, $(19,17),(27,24),(28,25),(35,31),(36,32),(37,33)$, $(38,34),(44,39),(45,40),(46,41),(47,42),(53,47)$, $(54,48),(55,49),,(56,50)$, and $(57,51)$ to resolve a toroidally localized ballooning mode. (Here $n, m$ are the toroidal and poloidal Fourier mode numbers, respectively.)

The large value of $n_{\max }(N=1)$ required, and the unusual spread in $n$ required in the basis set, will make these modes difficult to resolve using global eigenvalue codes (e.g., the simplifying phase factor method sometimes used in CAS3D studies [1] would not be appropriate). It is hoped that the Weyl formula estimate above will act as a guide in a future more extensive study using such a code. Physically, the large value of $n_{\max }$ suggests that toroidally localized ballooning modes in $\mathrm{H}-1 \mathrm{NF}$ should be subject to strong FLR stabilization.

We can also apply the same approach to the toroidally localized ballooning branches found in the large helical device (LHD) study [12]. From the plots in [12] we estimate $v_{3 \mathrm{D}} \sim 0.05$, which gives $n_{\max }(N=1) \approx 40$.

The ballooning calculations were carried out on the Australian National University Supercomputer Facility's Fujitsu VPP300 vector processor. We thank Dr. H.J. Gardner for providing the H-1 heliac vMEC input files and Dr. S.P. Hirshman for use of the VMEC equilibrium code. Some of this work was done while one of us (R. L.D.) was a visiting scientist at Princeton University Plasma Physics Laboratory, supported under US DOE contract No. DE-AC02-76CH0-3703. Useful conversations with Dr. M. Redi and Dr. A. H. Boozer are gratefully acknowledged.

*Permanent address: Research School of Physical Sciences \& Engineering, The Australian National University. Email address: robert.dewar@anu.edu.au

[1] A. H. Reiman et al., Plasma Phys. Rep. 23, 472 (1997).

[2] A. Reiman et al., Plasma Phys. Controlled Fusion 41, B273 (1999).

[3] G. H. Nielson et al., Phys. Plasmas 7, 1911 (2000).

[4] B. D. Blackwell, Bull. Am. Phys. Soc. 45, 289 (2000); Phys. Plasmas (to be published).

[5] I. B. Bernstein, E. A. Frieman, M. D. Kruskal, and R. M. Kulsrud, Proc. R. Soc. London A 244, 17 (1958).

[6] R. L. Dewar, J. Plasma Fusion Res. 73, 1123 (1997).

[7] R. L. Dewar and A.H. Glasser, Phys. Fluids 26, 3038 (1983).

[8] D. V. Anderson et al., Int. J. Supercomput. Appl. 4, 34 (1990).

[9] C. Schwab, Phys. Fluids B 5, 3195 (1993).

[10] R. L. Dewar, J. Manickam, R. C. Grimm, and M. S. Chance, Nucl. Fusion 21, 493 (1981); Nucl. Fusion 22, 307(E) (1982).

[11] W. A. Cooper, D. B. Singleton, and R. L. Dewar, Phys. Plasmas 3, 275 (1996); Phys. Plasmas 3, 3520(E) (1996).

[12] P. Cuthbert et al., Phys. Plasmas 5, 2921 (1998).

[13] E. Hameiri, Commun. Pure Appl. Math. 38, 43 (1985).

[14] A.E. Lifschitz, Magnetohydrodynamics and Spectral Theory (Kluwer, Dordrecht, The Netherlands, 1989), pp. 416-423.

[15] W. M. Nevins and L. D. Pearlstein, Phys. Fluids 31, 1988 (1988).

[16] M.C. Gutzwiller, Chaos in Classical and Quantum Mechanics, Interdisciplinary Applied Mathematics Series (Springer-Verlag, New York, 1990), Vol. 1, pp. 257-261.

[17] E. Ott, Chaos in Dynamical Systems (Cambridge University Press, Cambridge, U.K., 1993).

[18] W. M. Tang, R. L. Dewar, and J. Manickam, Nucl. Fusion 22, 1079 (1982).

[19] R. J. Hastie, P. J. Catto, and J. J. Ramos, Bull. Am. Phys. Soc. 45, 363 (2000).

[20] S. M. Hamberger, B. D. Blackwell, L. E. Sharp, and D. B. Shenton, Fusion Technol. 17, 123 (1990).

[21] S. P. Hirshman and O. Betancourt, J. Comput. Phys. 96, 99 (1991).

[22] P. Cuthbert and R. L. Dewar, Phys. Plasmas 7, 2302 (2000).

[23] W. A. Cooper and H. J. Gardner, Nucl. Fusion 34, 729 (1994). 